\title{
Two interacting particles in a spherical pore
}

Ignacio Urrutia, and Gabriela Castelletti

Citation: The Journal of Chemical Physics 134, 064508 (2011); doi: 10.1063/1.3544681

View online: https://doi.org/10.1063/1.3544681

View Table of Contents: http://aip.scitation.org/toc/jcp/134/6

Published by the American Institute of Physics

\section{Articles you may be interested in}

Structure, thermodynamic properties, and phase diagrams of few colloids confined in a spherical pore

The Journal of Chemical Physics 142, 244707 (2015); 10.1063/1.4923164

Three hard spheres in a spherical cavity

The Journal of Chemical Physics 135, 024511 (2011); 10.1063/1.3609796

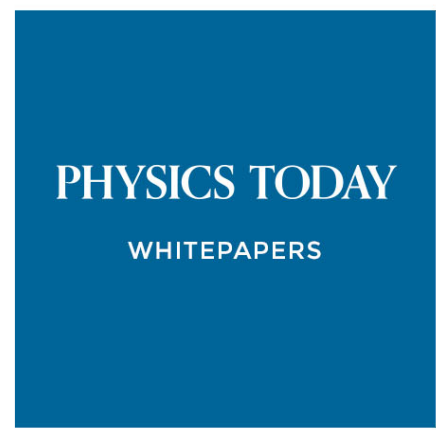




\title{
Two interacting particles in a spherical pore
}

\author{
Ignacio Urrutia ${ }^{1, a)}$ and Gabriela Castelletti ${ }^{2}$ \\ ${ }^{1}$ Consejo Nacional de Investigaciones Científicas y Técnicas, Argentina (CONICET) and Departamento de \\ Física, Comisión Nacional de Energía Atómica, Av. Gral. Paz 1499 (RA-1650) San Martín, Buenos Aires, \\ Argentina \\ ${ }^{2}$ Instituto de Astronomía y Física del Espacio (CONICET-UBA), Buenos Aires, Argentina and Facultad de \\ Ciencias Exactas y Naturales, Universidad de Buenos Aires, Argentina
}

(Received 2 November 2010; accepted 4 January 2011; published online 9 February 2011)

\begin{abstract}
In this work we analytically evaluate, for the first time, the exact canonical partition function for two interacting spherical particles into a spherical pore. The interaction with the spherical substrate and between particles is described by an attractive square-well and a square-shoulder potential. In addition, we obtain exact expressions for both the one particle and an averaged two particle density distribution. We develop a thermodynamic approach to few-body systems by introducing a method based on thermodynamic measures [I. Urrutia, J. Chem. Phys. 134, 104503 (2010)] for nonhard interaction potentials. This analysis enables us to obtain expressions for the pressure, the surface tension, and the equivalent magnitudes for the total and Gaussian curvatures. As a by-product, we solve systems composed of two particles outside a fixed spherical obstacle. We study the low density limit for a many-body system confined to a spherical cavity and a many-body system surrounding a spherical obstacle. From this analysis we derive the exact first order dependence of the surface tension and Tolman length. Our findings show that the Tolman length goes to zero in the case of a purely hard wall spherical substrate, but contains a zero order term in density for square-well and square-shoulder wall-fluid potentials. This suggests that any nonhard wall-fluid potential should produce a non-null zero order term in the Tolman length. (C) 2011 American Institute of Physics. [doi:10.1063/1.3544681]
\end{abstract}

\section{INTRODUCTION}

The properties of fluid systems in equilibrium are difficult to study in the framework of statistical mechanics theory of ensembles. Basically, the ensemble theory shows that the thermodynamic properties of a system composed of interacting particles are expressible in terms of a partition function. In a given ensemble some properties of the system are kept fixed. The canonical ensemble analyzed in this work, for instance, represents a system with a fixed number of particles, $N$, into a volume at constant temperature, $T$. In this ensemble the partition function $Q$ is an integral over the coordinates and moments of the particles. Due to the difficulties in deriving the analytical expression for $Q$, a direct approach to the thermodynamic properties of a fluid based on the partition function evaluation is often avoided in the literature.

The exact analytic expression for $Q$ has been recently presented for some few-body systems of hard spheres confined to cavities with simple geometries. ${ }^{1,2}$ From a theoretical point of view, the importance of these results is that they constitute the unique case for which an exact solution was found. Since its origin, the main interest of statistical mechanics theory of fluids was to deal with large systems, frequently involving the so-called thermodynamic limit. The theoretical development, however, shows that the initial interest on large homogeneous systems made gradually place to inhomogeneous ones. Most of the works in the literature devoted on fluids pay special attention to inhomogeneous systems and

\footnotetext{
${ }^{a)}$ Electronic mail: iurrutia@cnea.gov.ar.
}

particularly to analyze the properties of fluids under confinement. Indeed, a continue effort to implement the statistical mechanics recipes to increasingly smaller inhomogeneous fluid systems has been noticeable in the last decades. Similarly, the thermodynamic theory although developed earlier than statistical mechanics, was initially focused on large systems and gradually dropped to the smaller ones. In summary, these last statements highlight the importance of few-body inhomogeneous systems and show the convenience of studying them considering a statistical mechanics and a thermodynamic framework.

Up to present, all the exactly solved few-body systems in three dimensions concern two hard spheres (HS). The 2-HS system confined to hard cavities of various simple geometrical shapes was studied in the framework of the canonical ensemble. The analyzed shapes include the spherical cavity, ${ }^{2}$ the spherical cavity with a hard central core,${ }^{3}$ the cuboidal, the cylindrical and the spheroidal, cavities. ${ }^{1}$ A remarkable characteristic of HS systems is that their thermodynamics is driven by entropy. Indeed, in such systems the temperature does not play any interesting role and thus the energy is equal to that of the ideal gas; for this reason HS systems are commonly referred as athermal.

The square-well potential (SW) is a natural extension of the HS potential, which includes an attractive well and it is still simple enough to handle analytically. This potential was used to model the interaction between atoms in a simple fluid $^{4-7}$ as well as to simulate the effective interaction between colloidal particles. ${ }^{8}$ In this paper we present an exact study of a 2-SW system composed of particles confined to a SW 
spherical cavity (SWC), the so-called 2-SW-SWC. The analytical knowledge of the statistical mechanics properties of a system composed of two colloidal particles with short range potential constitutes a valuable contribution to the subject. We verify our findings by comparing them with the previously published numerical results for a similar system. ${ }^{9}$ We also include in our study the cases of square-shoulder potentials (SS) for both particle-particle and particle-substrate interactions. Other interesting characteristic of our work is that it focuses on solving exactly a spherically inhomogeneous fluid system of interacting particles. Some works that reflect the actual interest in spherically symmetric macroscopic systems are those related to the study of fluid confined to spherical cavities, and those in contact with a spherical particle. ${ }^{10,11}$ As we will show in our analysis, some properties of this macroscopic system related to the substrate-fluid surface tension and the Tolman length will be derived from the properties of the 2-SW-SWC system.

This paper is organized as follows: in Sec. II we described the analytical evaluation of the partition function for a system of two particles confined to a spherical pore, where the interactions are described by piecewise constant potentials. The 2-SW-SWC, 2-SW-SSC, 2-SS-SWC, and 2-SS-SSC systems are particular cases of the obtained solution. Additionally, we obtain analytically the one-body density distribution and an averaged two-body density distribution. In Sec. III we discuss the implementation of a thermodynamic scheme to evaluate the global properties of a few-body system and study the thermodynamic properties of a 2-SW-SWC system. The analytical correspondence between the properties of the 2-SW-SWC system and other few-body systems as well as between 2-SW-SWC and many-body systems composed of SW and SS particles is presented in Sec. IV. In this section we derive the first non-null term in a series expansion of the fluid-substrate surface tension and Tolman length in powers of the density. A summary of our work is presented in Sec. V.

\section{EVALUATION OF THE PARTITION FUNCTION}

We are interested in furthering the understanding of the thermodynamic properties of fluidlike small inhomogeneous systems of confined particles. Let us therefore consider a system composed of $N$ particles confined by an external potential $\varphi_{N}=\sum \varphi\left(\mathbf{r}_{i}\right)$, in which $\phi_{N}=\sum \phi\left(r_{i j}\right)$ represents the interaction between particles. The partition function of this system is

$Q=\Lambda^{-N D} Z=\Lambda^{-N D} \int \exp \left(-\beta \varphi_{N}\right) \exp \left(-\beta \phi_{N}\right) \prod_{i=1}^{N} d \mathbf{r}_{i}$,

where $Z$ is the configuration integral (CI), $\Lambda$ $=h /\left(2 \pi m k_{B} T\right)^{1 / 2}$ is the thermal de Broglie wavelength, $\beta=\left(k_{B} T\right)^{-1}$ is the inverse temperature, $k_{B}$ is the Boltzmann's constant, $T$ is the temperature, and $D$ is the dimension of the space. Such a system constitutes the general framework for our study. Even though our main interest is in the general system described by Eq. (1), we decided to reduce the complexity of the analysis by focusing on the study of the

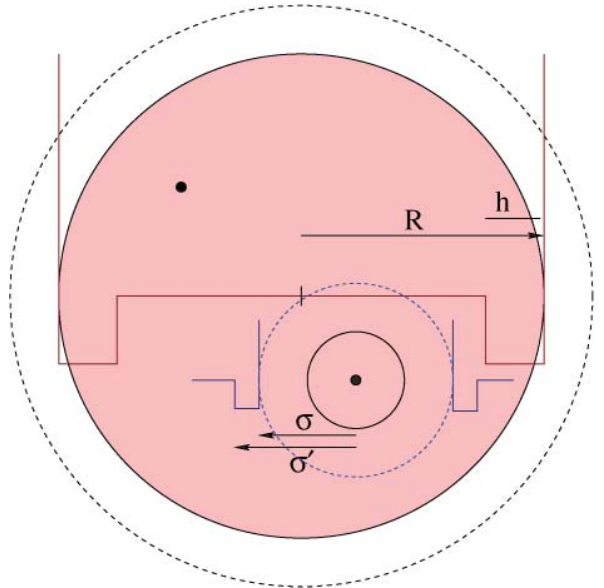

FIG. 1. A scheme of the 2-SW-SWC system showing the two square-well particles confined to a square-well spherical cavity.

smallest nonhard and nontrivial system composed of two interacting particles. Specifically, we consider the CI of the 2-SW-SWC system. The diameter or hard repulsion distance between particles is $\sigma$, while the radius of the empty cavity is $R+\sigma / 2$, with $R$ the effective radius of the pore. In Fig. 1 we draw the main characteristics of the 2-SW-SWC system and also indicate the lengths of the square wells $\sigma^{\prime}-\sigma$ and $h$. Taking into account this representation for the 2-SW-SWC system, we evaluate the canonical partition function $Q$ for the slightly more general case of piecewise constant interactions in $D$ dimensions. It is given by

$$
\begin{aligned}
& Q=\Lambda^{-2 D} Z=\Lambda^{-2 D} \iint e_{1} e_{2} e_{12} d \mathbf{r}_{1} d \mathbf{r}_{2}, \\
& e_{i} \equiv \exp \left[-\beta \varphi\left(r_{i}\right)\right]= \begin{cases}Y_{\mathbf{0}}, & \text { if } r_{i} \leq R-h, \\
Y_{1}, & \text { if } R-h<r_{i} \leq R, \\
0, & \text { if } r_{i}>R,\end{cases} \\
& e_{12} \equiv \exp \left[-\beta \phi\left(r_{12}\right)\right]= \begin{cases}X_{\mathbf{0}}, & \text { if } r_{12} \leq \sigma, \\
X_{1}, & \text { if } \sigma<r_{12} \leq \sigma^{\prime}, \\
X_{\mathbf{2}}, & \text { if } r_{12}>\sigma^{\prime} .\end{cases}
\end{aligned}
$$

Here, normal and bold numeric subindices are used to label particles and piecewise potential, respectively. The bold subindex will be also used below to label different functions and parameters, but this will not cause confusion because its meaning will be clear from the context. The Eq. (4) can also be rewritten as

$e_{12}=X_{\mathbf{0}}+\left(X_{1}-X_{\mathbf{0}}\right) \Theta\left(r_{12}-\sigma\right)+\left(X_{2}-X_{1}\right) \Theta\left(r_{12}-\sigma^{\prime}\right)$,

where $\Theta$ is the Heaviside function defined by: $\Theta(x)=1$ if $x \geq 0$ and $\Theta(x)=0$ otherwise. Note that at a given temperature $X_{\mathbf{i}}=\exp \left(-\beta \phi_{\mathbf{i}}\right)$ and $Y_{\mathbf{i}}=\exp \left(-\beta \varphi_{\mathbf{i}}\right)$ with $\phi_{\mathbf{i}}$ and $\varphi_{\mathbf{i}}$ constants, do not depend on coordinates and $X_{\mathbf{i}}, Y_{\mathbf{i}} \geq 0$. The SW particles are obtained by setting $X_{0}=0, X_{1}>1$, and $X_{2}=1$, while the SWC is obtained in the case $Y_{0}=1$ and $Y_{1}>1$. On the other hand, the square-shoulder potentials use $0<X_{1}<$ 1 and/or $0<Y_{1}<1$. In Fig. 2 we display all the possible combinations using a general notation in terms of $X$ and $Y$ 


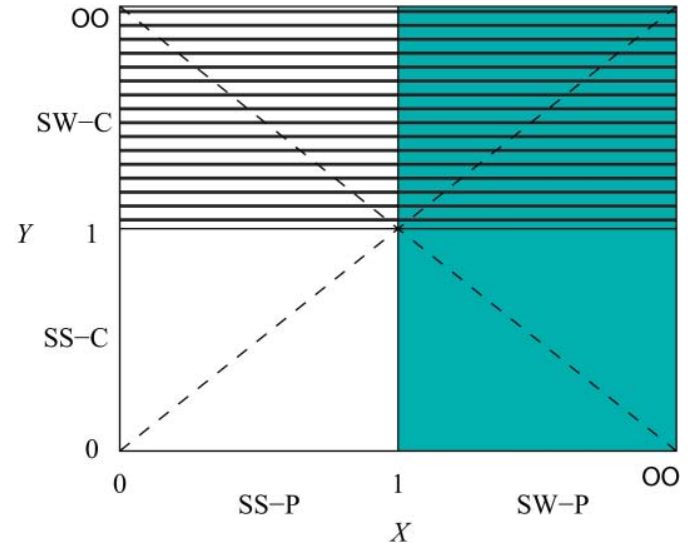

FIG. 2. Phase space diagram in the $X, Y$ parameters for SW and SS potentials. The vertical line $X=1$ corresponds to the HS system, while the horizontal line $Y=1$ corresponds to the HC. The central point with $X=1$ and $Y=1$ corresponds to HS particles confined to a HC. Systems with $\lambda=1$ and $\lambda=-1$ are represented with dashed lines for reference.

instead of $X_{1}$ and $Y_{1}$. In this representation, the $X$ and $Y$ axis correspond to the inter-particle potential properties and the particle-substrate potential, respectively. The system can be adequately described by a point in this square by assuming a fixed potential and temperature. For fixed values of $\phi_{1}$ and $\varphi_{1}$, an increase in $T$ produces the motion of the system point on the curve $Y=X^{\lambda}\left(\lambda=\varphi_{1} / \phi_{1}\right)$ toward the central portion of the square. These trajectories never cross the lines $X=1$ and $Y=1$. The spherical pore exerts a simple hard potential when $Y=1$, thus, in this case we deal with a hard cavity (HC).

Now, we return to Eq. (2). Using the center of the pore together with the relative distance between particles as the integration variables, and integrating on the coordinates of the pore center, the Eq. (2) can be written as

$$
\begin{aligned}
Z= & \Omega(D) \int_{0}^{2 R} e_{12}(r)\left[\left(Y_{\mathbf{0}}-Y_{\mathbf{1}}\right)^{2} \mathcal{J}_{\mathbf{1}}\right. \\
& \left.+2 Y_{\mathbf{1}}\left(Y_{\mathbf{0}}-Y_{\mathbf{1}}\right) \mathcal{J}_{\mathbf{2}}+Y_{\mathbf{1}}^{2} \mathcal{J}_{\mathbf{3}}\right] r^{D-1} d r
\end{aligned}
$$

where $\Omega(D)$ represents the solid angle. To write down $\mathcal{J}_{1}, \mathcal{J}_{2}$, and $\mathcal{J}_{\mathbf{3}}$ we introduce the function $J\left(r, R_{\mathbf{1}}, R_{\mathbf{2}}\right)$ for the overlap volume between two spheres of radii $R_{\mathbf{1}}$ and $R_{\mathbf{2}}$ (being $R_{1} \geq R_{2}$ ) whose centers are separated by a distance $r \geq 0$,

$$
J\left(r, R_{\mathbf{1}}, R_{\mathbf{2}}\right) \equiv \begin{cases}S p\left(R_{\mathbf{2}}\right), & r \leq R_{\mathbf{1}}-R_{\mathbf{2}} \\ I\left(r, R_{\mathbf{1}}, R_{\mathbf{2}}\right), & \text { otherwise } \\ 0, & r>R_{\mathbf{1}}+R_{\mathbf{2}}\end{cases}
$$

where $\operatorname{Sp}(R)$ is the volume of the sphere of radius $R$, while $I\left(r, R_{\mathbf{1}}, R_{\mathbf{2}}\right)$ is the overlap volume in the partially overlapping configuration, which is symmetric through the permutation $R_{\mathbf{1}} \leftrightarrow R_{\mathbf{2}}$. We complete the definition given in Eq. (7) by setting $J\left(r, R_{\mathbf{1}}, R_{\mathbf{2}}\right) \equiv 0$ for the unphysical values $R_{\mathbf{2}} \leq 0$. For the special case $R_{\mathbf{1}}=R_{\mathbf{2}}$, we use the shortcut expressions $J\left(r, R_{\mathbf{1}}\right) \equiv J\left(r, R_{\mathbf{1}}, R_{\mathbf{1}}\right)$ and $I\left(r, R_{\mathbf{1}}\right) \equiv I\left(r, R_{\mathbf{1}}, R_{\mathbf{1}}\right)$ related to each other by

$$
J\left(r, R_{\mathbf{1}}\right)= \begin{cases}I\left(r, R_{\mathbf{1}}\right), & 0<r \leq 2 R_{\mathbf{1}}, \\ 0, & r>2 R_{\mathbf{1}},\end{cases}
$$

TABLE I. Summary of all the possible order relations between the values of the length parameters that are relevant in Eq. (6). Magnitudes increase from left to right.

\begin{tabular}{ccccccc}
\hline \hline 1 & $h$ & $\sigma$ & $\sigma^{\prime}$ & $2(R-h)$ & $2 R-h$ & $2 R$ \\
2 & $h$ & $\sigma$ & $2(R-h)$ & $\sigma^{\prime}$ & $2 R-h$ & $2 R$ \\
3 & $h$ & $\sigma$ & $2(R-h)$ & $2 R-h$ & $\sigma^{\prime}$ & $2 R$ \\
4 & $h$ & $\sigma$ & $2(R-h)$ & $2 R-h$ & $2 R$ & $\sigma^{\prime}$ \\
5 & $h$ & $2(R-h)$ & $\sigma$ & $\sigma^{\prime}$ & $2 R-h$ & $2 R$ \\
6 & $h$ & $2(R-h)$ & $\sigma$ & $2 R-h$ & $\sigma^{\prime}$ & $2 R$ \\
7 & $h$ & $2(R-h)$ & $\sigma$ & $2 R-h$ & $2 R$ & $\sigma^{\prime}$ \\
8 & $h$ & $2(R-h)$ & $2 R-h$ & $\sigma$ & $\sigma^{\prime}$ & $2 R$ \\
9 & $h$ & $2(R-h)$ & $2 R-h$ & $\sigma$ & $2 R$ & $\sigma^{\prime}$ \\
10 & $h$ & $2(R-h)$ & $2 R-h$ & $2 R$ & $\sigma$ & $\sigma^{\prime}$ \\
11 & $2(R-h)$ & $h$ & $\sigma$ & $\sigma^{\prime}$ & $2 R-h$ & $2 R$ \\
12 & $2(R-h)$ & $h$ & $\sigma$ & $2 R-h$ & $\sigma^{\prime}$ & $2 R$ \\
13 & $2(R-h)$ & $h$ & $\sigma$ & $2 R-h$ & $2 R$ & $\sigma^{\prime}$ \\
14 & $2(R-h)$ & $h$ & $2 R-h$ & $\sigma$ & $\sigma^{\prime}$ & $2 R$ \\
15 & $2(R-h)$ & $h$ & $2 R-h$ & $\sigma$ & $2 R$ & $\sigma^{\prime}$ \\
16 & $2(R-h)$ & $h$ & $2 R-h$ & $2 R$ & $\sigma$ & $\sigma^{\prime}$ \\
17 & $\sigma$ & $h$ & $\sigma^{\prime}$ & $2(R-h)$ & $2 R-h$ & $2 R$ \\
18 & $\sigma$ & $h$ & $2(R-h)$ & $\sigma^{\prime}$ & $2 R-h$ & $2 R$ \\
19 & $\sigma$ & $h$ & $2(R-h)$ & $2 R-h$ & $\sigma^{\prime}$ & $2 R$ \\
20 & $\sigma$ & $h$ & $2(R-h)$ & $2 R-h$ & $2 R$ & $\sigma^{\prime}$ \\
21 & $2(R-h)$ & $\sigma$ & $h$ & $\sigma^{\prime}$ & $2 R-h$ & $2 R$ \\
22 & $2(R-h)$ & $\sigma$ & $h$ & $2 R-h$ & $\sigma^{\prime}$ & $2 R$ \\
23 & $2(R-h)$ & $\sigma$ & $h$ & $2 R-h$ & $2 R$ & $\sigma^{\prime}$ \\
24 & $\sigma$ & $2(R-h)$ & $h$ & $\sigma^{\prime}$ & $2 R-h$ & $2 R$ \\
25 & $\sigma$ & $2(R-h)$ & $h$ & $2 R-h$ & $\sigma^{\prime}$ & $2 R$ \\
26 & $\sigma$ & $2(R-h)$ & $h$ & $2 R-h$ & $2 R$ & $\sigma^{\prime}$ \\
27 & $\sigma$ & $\sigma^{\prime}$ & $h$ & $2(R-h)$ & $2 R-h$ & $2 R$ \\
28 & $\sigma$ & $\sigma^{\prime}$ & $2(R-h)$ & $h$ & $2 R-h$ & $2 R$ \\
29 & $\sigma$ & $2(R-h)$ & $\sigma^{\prime}$ & $h$ & $2 R-h$ & $2 R$ \\
30 & $2(R-h)$ & $\sigma$ & $\sigma^{\prime}$ & $h$ & $2 R-h$ & $2 R$ \\
\hline \hline & & & & & &
\end{tabular}

and we set $J\left(r, R_{\mathbf{1}}\right) \equiv 0$ for $R_{\mathbf{1}} \leq 0$. The expressions of $\Omega(D)$, $J\left(r, R_{\mathbf{1}}, R_{\mathbf{2}}\right)$ as well as $J\left(r, R_{\mathbf{1}}\right)$ for any dimension can be find in the literature. ${ }^{2,3}$ From Eqs. (6) to (8) we obtain

$$
\begin{aligned}
& \mathcal{J}_{1}=J(r, R-h), \\
& \mathcal{J}_{2}=J(r, R, R-h), \\
& \mathcal{J}_{3}=J(r, R) .
\end{aligned}
$$

Upon inspecting the set of equations from Eqs. (6) to (11) it is evident the discontinuity in the integrand in Eq. (6) at $r$ $=\left\{\sigma, \sigma^{\prime}\right\}$ and in its second derivative at $r=\{h, 2(R$ $-h), 2 R-h\}$. The unanalycities of this integrand produce a nonanalytical partition function that spreads in several analytic branches. We find that depending on the order relation between the values of $\left\{\sigma, \sigma^{\prime}, h, 2(R-h), 2 R-h, 2 R\right\}$ a different analytic branch is yielded. These magnitudes satisfy the following inequalities: $0 \leq\{h, 2(R-h)\} \leq 2 R$ $-h \leq 2 R$ and $0 \leq \sigma \leq \sigma^{\prime}$. From the combination of the two sets of inequalities it is possible to distinguish 30 different cases, which are summarized in Table I.

For further progress we restrict the analysis to three dimensions, $D=3$, so $\Omega(3)=4 \pi, S p\left(R_{1}\right)=(4 \pi / 3) R_{1}^{3}$,

$$
I\left(r, R_{\mathbf{1}}\right)=\frac{\pi}{12}\left(2 R_{\mathbf{1}}-r\right)^{2}\left(r+4 R_{\mathbf{1}}\right)
$$




$$
\begin{aligned}
I\left(r, R_{\mathbf{1}}, R_{\mathbf{2}}\right)= & \frac{\pi}{12 r}\left(R_{\mathbf{1}}+R_{\mathbf{2}}-r\right)^{2}\left(r^{2}-3\left(R_{\mathbf{1}}-R_{\mathbf{2}}\right)^{2}\right. \\
& \left.+2 r\left(R_{\mathbf{1}}+R_{\mathbf{2}}\right)\right) .
\end{aligned}
$$

Functions $I\left(r, R_{1}\right)$ and $I\left(r, R_{1}, R_{2}\right)$ for integer values of $D$ were published in Refs. 2 and 3. It is worthwhile to note that for each analytic domain the integrand in Eq. (6) is a polynomial in the variables $\left\{r, R, h, \sigma, \sigma^{\prime}\right\}$. The integrals $\mathcal{L}_{\mathbf{i}}=\mathcal{I}\left[\mathcal{J}_{\mathbf{i}}\right] \equiv 4 \pi \int_{0}^{s} \mathcal{J}_{\mathbf{i}} r^{2} d r$ for $\mathbf{i}=\mathbf{1}, \mathbf{2}, \mathbf{3}$ are given by

$$
\mathcal{L}_{\mathbf{1}}(s)=\mathcal{I}[J(r, R-h)], \quad \mathcal{L}_{\mathbf{3}}(s)=\mathcal{I}[J(r, R)],
$$

$$
\begin{aligned}
& \mathcal{I}\left[J\left(r, R_{\mathbf{1}}\right)\right]=\left(\frac{4 \pi}{3}\right)^{2} \begin{cases}2^{-5} s^{3}\left(s^{3}-18 s R_{\mathbf{1}}^{2}+32 R_{\mathbf{1}}^{3}\right), & \text { if } 0 \leq s \leq 2 R_{\mathbf{1}}, \\
R_{\mathbf{1}}^{6}, & \text { if } s>2 R_{\mathbf{1}},\end{cases} \\
& \mathcal{L}_{\mathbf{2}}(s)=\left(\frac{4 \pi}{3}\right)^{2} \begin{cases}s^{3}(R-h)^{3}, & \text { if } 0 \leq s \leq h, \\
2^{-5}\left[s^{6}-s^{4} 9\left(h^{2}-2 h R+2 R^{2}\right)-s^{2} 9 h^{2}(2 R-h)^{2}\right. & \left.s^{3} 16(2 R-h)\left(h^{2}-h R+R^{2}\right)+h^{4}\left(h^{2}-6 h R+6 R^{2}\right)\right], \\
(R-h)^{3} R^{3}, & \text { else, }\end{cases} \\
& \hline
\end{aligned}
$$

Note that the functions $\mathcal{L}_{\mathbf{i}}(s)$ are also polynomials but in the variables $\{s, R, h\}$. The same behavior is also valid for the equivalent problem in any odd dimension. ${ }^{3}$ Finally, we obtain the following expression for the $\mathrm{CI}$

$$
\begin{aligned}
Z= & \left(X_{\mathbf{0}}-X_{\mathbf{1}}, X_{\mathbf{1}}-X_{\mathbf{2}}, X_{\mathbf{2}}\right) \mathbf{K}\left[\begin{array}{ccc}
\mathcal{L}_{\mathbf{1}}(\sigma) & \mathcal{L}_{\mathbf{2}}(\sigma) & \mathcal{L}_{\mathbf{3}}(\sigma) \\
\mathcal{L}_{\mathbf{1}}\left(\sigma^{\prime}\right) & \mathcal{L}_{\mathbf{2}}\left(\sigma^{\prime}\right) & \mathcal{L}_{\mathbf{3}}\left(\sigma^{\prime}\right) \\
\mathcal{L}_{\mathbf{1}}(2 R) & \mathcal{L}_{\mathbf{2}}(2 R) & \mathcal{L}_{\mathbf{3}}(2 R)
\end{array}\right] \\
& \times\left(\begin{array}{c}
\left(Y_{\mathbf{0}}-Y_{\mathbf{1}}\right)^{2} \\
2 Y_{\mathbf{1}}\left(Y_{\mathbf{0}}-Y_{\mathbf{1}}\right) \\
Y_{1}^{2}
\end{array}\right) .
\end{aligned}
$$

Here $\mathbf{K}$ is a $3 \times 3$ matrix that considers all the different ways in which the parameters $\left\{\sigma, \sigma^{\prime}, 2 R\right\}$ can be arranged in increasing order once their values were fixed. For $\sigma^{\prime} \leq 2 R, \mathbf{K}$ is given by the identity matrix, while

$$
\begin{aligned}
& \mathbf{K}=\left[\begin{array}{lll}
1 & 0 & 0 \\
0 & 0 & 1 \\
0 & 0 & 1
\end{array}\right] \text { for } \sigma \leq 2 R<\sigma^{\prime}, \\
& \mathbf{K}=\left[\begin{array}{lll}
0 & 0 & 1 \\
0 & 0 & 1 \\
0 & 0 & 1
\end{array}\right] \text { for } 0 \leq 2 R<\sigma .
\end{aligned}
$$

We introduce the shortcuts: $\mathbf{L}$ for the matrix in Eq. (17), $\mathbf{X}$ and $\mathbf{Y}^{2}$ for the row vectors related to $X_{\mathbf{i}}$ and $Y_{\mathbf{i}}$ values, respectively. We combine them with standard vectorial notation to obtain

$$
Z=\mathbf{X} \cdot \mathbf{K} \cdot \mathbf{L} \cdot \mathbf{Y}^{2},
$$

where $\left(\mathbf{Y}^{2}\right)^{\dagger}$ is the transposed of the matrix $\mathbf{Y}^{2}$. This is an explicit and compact expression for the $\mathrm{CI}$ in which the temperature dependence is separated from the dependence on the characteristic lengths $\left\{\sigma, \sigma^{\prime}, h, R\right\}$. From Eq. (17), it is clear that the generalization to more complex piecewise constant potentials is straightforward. We have checked that our results for the special case of the 2-HS-SWC are consistent with the analytical results found by McQuarrie and Rowlinson. ${ }^{12}$ In addition, we introduce two relevant distribution functions: the one-body distribution function $\rho(\mathbf{r})$ and a kind of averaged pair distribution function $\bar{g}(\mathbf{r})$ in which the position of the pore center was integrated. ${ }^{2}$ Both distribution functions can be defined in terms of the two-body density distribution $\rho_{2}\left(\mathbf{r}_{1}, \mathbf{r}_{2}\right)$ (for further details about this function see for example Ref. 13),

$$
\begin{aligned}
& \rho(\mathbf{r})=(N-1)^{-1} \int \rho_{2}\left(\mathbf{r}, \mathbf{r}_{2}\right) d \mathbf{r}_{2}, \\
& \bar{g}(\mathbf{r}) \equiv \frac{1}{2} \int \rho_{2}\left(\mathbf{r}+\mathbf{r}_{2}, \mathbf{r}_{2}\right) d \mathbf{r}_{2},
\end{aligned}
$$

where $\rho(\mathbf{r})$ and $\bar{g}(\mathbf{r})$ are normalized to $N$ and $N(N-1) / 2$, respectively. Note that Eq. (21) is essentially the integrand in Eq. (6). Explicit expressions for the two distributions associated with the system studied in the current work are given by

$$
\begin{gathered}
\rho(r)=2 Z^{-1} e_{1}(r) \mathbf{X} \cdot \mathbf{K} \cdot \mathbf{J} \cdot \mathbf{Y}^{\dagger}, \\
\mathbf{J}=\left[\begin{array}{cc}
J(r,\{R-h, \sigma\}) & J(r,\{R, \sigma\}) \\
J\left(r,\left\{R-h, \sigma^{\prime}\right\}\right) & J\left(r,\left\{R, \sigma^{\prime}\right\}\right) \\
S p(R-h) & S p(R)
\end{array}\right], \\
\bar{g}(r)=Z^{-1} e_{12}(r)\left(\left(Y_{\mathbf{0}}-Y_{\mathbf{1}}\right)^{2} \mathcal{J}_{\mathbf{1}}\right. \\
\left.+2 Y_{\mathbf{1}}\left(Y_{\mathbf{0}}-Y_{\mathbf{1}}\right) \mathcal{J}_{\mathbf{2}}+Y_{\mathbf{1}}^{2} \mathcal{J}_{\mathbf{3}}\right),
\end{gathered}
$$

where $\mathbf{Y}=\left(Y_{0}-Y_{1}, Y_{1}\right)$ and $J\left(r,\left\{R_{1}, R_{2}\right\}\right) \equiv J(r, \operatorname{Max}$ $\left.\left(R_{1}, R_{2}\right), \operatorname{Min}\left(R_{1}, R_{2}\right)\right)$ is an extension of Eq. (7). It is worthwhile that for $R>h+\sigma^{\prime} / 2$, a homogeneous density plateau is developed in the central region of the cavity with $0 \leq r$ $\leq R-h-\sigma^{\prime} / 2$. A peripheral density plateau is developed if $h>2 \sigma^{\prime}$ in the region $R-h+\sigma^{\prime} \leq r \leq R-\sigma^{\prime}$. The density in the plateau regions is given by

$$
\begin{aligned}
\rho_{h}= & 2 Z^{-1} e_{1}\left\{X_{2}\left[Y_{1} S p(R)+\left(Y_{0}-Y_{1}\right) S p(R-h)\right]\right. \\
& \left.+Y_{0}\left[\left(X_{1}-X_{2}\right) S p\left(\sigma^{\prime}\right)+\left(X_{0}-X_{1}\right) S p(\sigma)\right]\right\},
\end{aligned}
$$

where the dependence on the position comes from Eq. (3), i.e., $e_{1}=Y_{0}$ for the central plateau and $e_{1}=Y_{1}$ for the peripheral one. The homogeneous density in both cases can be simplified by introducing the CI corresponding to the one particle system, $Z_{(1)}=Y_{1} S p(R)+\left(Y_{0}\right.$ 
$\left.-Y_{1}\right) S p(R-h)$, and the first cluster integral of the homogeneous SW-particle system, $2 b_{2} \equiv-\int\left(e_{12}-1\right) d \mathbf{r}_{12}$ $=\left(X_{1}-X_{0}\right) \operatorname{Sp}(\sigma)+\left(X_{2}-X_{1}\right) \operatorname{Sp}\left(\sigma^{\prime}\right)$ giving

$$
\rho_{h}=2 Z^{-1}\left(e_{1} X_{2} Z_{(1)}-e_{1}^{2} 2 b_{2}\right) \text {. }
$$

Note that in our convention $b_{2}>0$ for HS. Finally, the pressure in the homogeneous regions may be evaluated using the procedure developed in Ref. 1 . We thus find

$$
\beta P_{h}=\rho_{h}+Z^{-1} e_{1}^{2} 2 b_{2}=2 Z^{-1}\left(e_{1} X_{2} Z_{(1)}-e_{1}^{2} b_{2}\right) .
$$

The compressibility factor in the homogeneous density region provides a local equation of state (EOS) that describes the local properties in the plateaus. It is given by

$$
\frac{\beta P_{h}}{\rho_{h}}=1+\frac{1}{2} \frac{b_{2}}{X_{2} / e_{1} \bar{\rho}^{-1}-b_{2}},
$$

where $\bar{\rho}=2 / Z_{(1)}$ is the rough density. Equation (28) for $X_{2} / e_{1}=1$ has been previously obtained for both, the case of 2-HS system confined to an arbitrary general geometry ${ }^{1}$ and for 2-HS in a hard wall spherical confinement in $D$ dimensions. ${ }^{3}$ We conclude that Eq. (28) can be also applied in 2-SW in other nonspherical SW confinement and further, to more general finite range potentials.

\section{THERMODYNAMICS OF A FEW-BODY SYSTEM UNDER CONFINEMENT}

The aim of this section is to analyze the thermodynamic behavior of few-body confined systems. Our main interest is the macroscopic description of small systems. It means that we are not interested in the peculiarities of the two-body system, but our goal is to find the EOS that describes the global properties of a few-body fluid system without any restriction on the number of particles. It is important to emphasize that a few-body system is far away from the thermodynamic limit of $N$-body systems with $N \rightarrow \infty$, therefore, the thermodynamic description developed below does not involve such limit. Also, the different ensemble representations for a fewbody system are not equivalent to each other. Of course, it does not produce any contradiction with macroscopic thermodynamics where the question of equivalence between different ensembles in the thermodynamic limit is secured. We assume that the system in which we are concerned, characterized by some external constraints, is well described by a certain Gibbsian ensemble and we will analyze the properties of this ensemble representation. Therefore, for a small system under the external constraints of constant temperature and number of particles, the appropriate thermodynamic analysis is one in which $N$ and $T$ are external parameters ${ }^{14}$ and thus, the ensemble representation to be used is the canonical one.

The pertinence of the thermodynamic theory to small systems was previously recognized by several authors, see e.g., Ref. 14. Even though the thermodynamic analysis of fewbody systems may seem controversial, we assert that it is possible to apply the first law of thermodynamic provided that all assumptions concerning the extensivity of the energy and entropy are avoided. The study presented in Ref. 1 supports this assertion and the overall thermodynamic approach that we will use here. In that work it was assumed that thermo- dynamics has the Simplicity, Universality and Size Invariance (SUSI) attributes, which implies that a consistent thermodynamic treatment of systems with large, many, and few number of particles is possible using a basic small set of macroscopic quantities. This exact thermodynamic approach allows an unified study of small (and inhomogeneous) systems constrained in cavities with different geometries and leads to results which are consistent with several exact relations as the wall theorem, which is a posteriori support of the assumptions.

\section{A. Free energy and work}

We would like to draw attention to an unsolved problem in equilibrium statistical mechanics. In effect, at first glance may seem surprising that even when we know the exact partition function of an inhomogeneous fluid system, its thermodynamic properties are not revealed. Our knowledge about the partition function comes from the exact evaluation of the integral in Eq. (1). Since the integrand and the limits of evaluation are functions of $T$ and of some set of mechanical parameters $\mathbf{X}$, the solution of that integral is expressed in terms of a function $Q(T, \mathbf{X})$. In the present thermodynamic approach we assume that the system has only one independent mechanical coordinate denoted by $R$. In classical thermodynamics it is well known that the free energy $F$, the energy $U$, the entropy $S$, and the chemical potential $\mu$ are related to each other by

$$
\begin{aligned}
& F=U-T S, \\
& d U=T d S-\mathcal{F}_{R} d R, \\
& d F=-S d T-\mathcal{F}_{R} d R, \\
& \mu_{N} \equiv F(N)-F(N-1) .
\end{aligned}
$$

Equations (30) and (31) involve infinitesimal variations of the magnitudes in a reversible transformation. From Eq. (31), it is clear that

$$
\mathcal{F}_{R}=-\partial_{R} F, \quad S=-\partial_{T} F, \quad U=\partial_{\beta} \beta F,
$$

where the partial derivatives are taken with respect to the variables $T$ (or $\beta$ ) and $R$ while the other parameters of the system are kept fixed in the calculation. Since $\mathcal{F}_{R}$ is the scalar force between the substrate and the fluid, the mechanical work done by the fluid due to an infinitesimal reversible change of the parameter $R$ is $d w=\mathcal{F}_{R} d R$. The total work in going from state$a$ to state- $b$ through an isothermal reversible transformation is

$$
w_{a b}=\int_{a}^{b} \mathcal{F}_{R} d R=F\left(R_{a}\right)-F\left(R_{b}\right) .
$$

Herefrom we assume that the other characteristic lengths and the energy parameters related to the interaction potentials are kept fixed when the $R$-piston is moved. The adopted definition for the chemical potential in Eq. (32) with $N \geq 1$ and $\mu_{0}=F(0)=0$ means that $\mu_{N}$ is the free energy increase which results from the addition of one particle to the $N-1$ particle system. We now establish the connection between thermodynamics and statistical mechanics via the usual relation 


$$
\beta F=-\ln (Q) \text {. }
$$

The scalar force and the energy may be also expressed as ensemble averages. Assuming spherical symmetry and relating $R$ to the radius of a divergent substrate potential, we find

$$
\begin{aligned}
\beta \mathcal{F}_{R} \equiv & -\beta\left\langle\frac{d}{d \mathbf{r}} \varphi_{N} \cdot \hat{\mathbf{r}}\right\rangle=-\beta \int \frac{d \varphi}{d r} \rho(r) d \mathbf{r}, \\
\beta U \equiv & \beta\left\langle E_{K}+\varphi_{N}+\phi_{N}\right\rangle=3 N / 2+\beta \\
& \times\left[\int \varphi(r) \rho(r) d \mathbf{r}+\int \phi(r) \bar{g}(r) d \mathbf{r}\right],
\end{aligned}
$$

where $E_{K}$ is the kinetic energy and \langle\rangle refers to the ensemble average of the physical quantities.

Concerning the 2-SW-SWC system, the right hand side in Eqs. (36) and (37) involves integrands which are piecewise polynomial functions. The evaluation of these integrals provide expressions for $\mathcal{F}_{R}$ and $U$, which are similar to those in Eqs. (19) and (17). For instance, from Eq. (36) we obtain a simple expression for $\mathcal{F}_{R}$,

$$
\begin{aligned}
\beta \mathcal{F}_{R}= & 4 \pi\left[Y_{1} R^{2} \rho(R)+\left(Y_{0}-Y_{1}\right)(R-h)^{2}\right. \\
& \times \rho(R-h) \Theta(R-h)] .
\end{aligned}
$$

In the thermodynamic description of a system, it is customary to assume that the analytical knowledge of $F$ permits to evaluate all the thermodynamic properties of the system. This statement may be ensured for extended homogeneous systems but not for confined inhomogeneous systems. As a simple example, in this work we evaluated $F(T, R)$, but to derive the thermodynamic pressure of the system it is also necessary to add the notion of volume $V$ of the system and establish the dependence of $F$ on $V$.

\section{B. Thermodynamic measures}

In order to complete the thermodynamic description of few-body systems it is necessary to define a suitable set of variables of state conforming with SUSI requirements. We introduce a set of thermodynamics measures $\mathbf{M}$ that allows us to obtain an exact description for few-body analytically solved systems. It is expected that a good choice of $\mathbf{M}$ yields thermodynamic properties in good agreement with well-known previously established results. Homogeneous fluids are typically described using the volume as measure, $\mathbf{M}=\{V\}$, while $\mathbf{M}=\{V, A\}$ with $A$ the surface area is often used for several authors in the study of inhomogeneous systems with interfaces [e.g., see Eq. (7) in Ref. 10]. The usual analysis of an ideal gas shows that the expected behavior for noninteracting particles is well described by a set $\mathbf{M}=\{V\}$, with $P=-\partial_{V} F(\mathbf{M})$ that should be compatible with the pressure of the system. A similar idea applies for the surface area of the substrate and the wall-fluid surface tension $\gamma$. We establish the relation between $\mathbf{M}$ and Eqs. (29), (31), and (35) on the basis of the exact description of the mechanical work

$$
w_{a b}=-\int_{a}^{b} \nabla_{\mathbf{M}} F \cdot d \mathbf{M}=-\int_{a}^{b} \mathbf{m} \cdot d \mathbf{M}
$$

where $\mathbf{m} \equiv \nabla_{\mathbf{M}} F$ and $d \mathbf{M}=\partial_{R} \mathbf{M} d R$. For the spherical symmetric system we find

$$
\partial_{R} F=\mathbf{m} \cdot \partial_{R} \mathbf{M}=\left.\sum_{j} \partial_{R} M_{j} \frac{\partial F}{\partial M_{j}}\right|_{T, \mathbf{M}-M_{j}},
$$

where the subscript $\mathbf{M}-M_{j}$ means that all the measures except the $j$-component are kept constant in the partial derivative. We consider a set of measures that includes volume and area measures $\mathbf{M}=\left\{V, A, M_{3}, \ldots\right\}$. The selection of $\mathbf{M}$ together with a rule that identifies the dependencies of the free energy $F$ on $\mathbf{M}$ [i.e., $F \rightarrow F(T, N, \mathbf{M})$ or $Z \rightarrow$ $Z(T, N, \mathbf{M})]$, allow us to find the so-called intensive thermodynamic magnitudes

$$
\begin{aligned}
& -P=\left.\frac{\partial F}{\partial V}\right|_{T, \mathbf{M}-V}, \\
& \gamma=\left.\frac{\partial F}{\partial A}\right|_{T, \mathbf{M}-A}, \\
& C_{M_{i}}=\left.\frac{\partial F}{\partial M_{i}}\right|_{T, \mathbf{M}-M_{i}},
\end{aligned}
$$

where $i \geq 3$. Clearly, Eqs. (41)-(43) are strongly dependent on the adopted $\mathbf{M}$.

Turning to the thermodynamic analysis of the 2-SWSWC system and following, ${ }^{1}$ we write the CI of this system as

$$
Z=Z_{(1)}^{2}-2 Z_{(1)} b_{2} \text { (pore). }
$$

The first term in the last equation reproduces the expected behavior of an ideal gas composed of two noninteracting particles $\left[b_{2}\right.$ (pore) $\left.=0\right]$ suggesting that $Z_{(1)}$ is a good measure of the system volume. Accordingly, we define the volume measure $V \equiv Z_{(1)}=\int e_{1}(r) d \mathbf{r}$ and obtain an expression for the pressure-for-work ${ }^{1}$

$$
P_{w}=\mathcal{F}_{R}\left(\frac{\partial V}{\partial R}\right)^{-1},
$$

which gives $d w=P_{w} d V$ for the total work. The $\beta P_{w}(R)$ function defined in Eq. (45) is plotted, for the case of the 2SS-HC system (i.e., $Y=1$ and $0<X<1$ ), in Figs. 1 and 2 of Ref. 9. That figure shows a van der Waals loop developed in the range $0.5<R / \sigma<2$ which becomes deeper with increasing $\phi_{1}$ and decreasing $T$ or $\sigma^{\prime}-\sigma$.

The last term in Eq. (44) may be decomposed by expanding

$$
Z_{(1)} b_{2}(\text { pore })=V b_{2}-a_{2} A+c_{2, \mathrm{~J}} \mathrm{~J}+c_{2, \mathrm{~K}} \mathrm{~K} .
$$

Here, we have introduced the measures for the area $A$ and the extensivelike mean and Gaussian curvatures $\mathrm{J}, \mathrm{K}$, respectively. The selection of a convenient definition for $A, \mathrm{~J}$, and $\mathrm{K}$ is in fact a nontrivial problem, which requires that geometrical and physical aspects to be considered. We have analyzed several possible definitions and select $A \equiv \int\left|\nabla e_{1}(\mathbf{r})\right| d \mathbf{r}$, which for a spherical confinement is reduced to $A=4 \pi \int r^{2}\left|\partial_{r} e_{1}\right| d r$. For this confinement, we select $\mathrm{J}=-4 \pi \int r^{2} j(r) \partial_{r} e_{1} d r$ and $\mathrm{K}=(1 / 2) 4 \pi \int r^{2} k(r)\left|\partial_{r} e_{1}\right| d r$, with $\quad j(r)=2 r^{-1} \quad$ and 
$k(r)=r^{-2}$. We then construct the set of thermodynamic measures $\mathbf{M}=\{V, A, \mathrm{~J}, \mathrm{~K}\}$. The definitions of $\mathrm{J}$ and $\mathrm{K}$ for nonspherical cavities with hard wall interaction potentials could be obtained by using the shape operator defined in the context of differential geometry of surfaces. In order to simplify the notation, we reduce the number of free parameters by setting $X_{0}=0, X_{1}=X=\exp \left(-\beta \phi_{1}\right), X_{2}=1, Y_{0}=1$, and $Y_{1}=Y=\exp \left(-\beta \varphi_{1}\right)$. Now, only two characteristic energies are present in the calculation: the substrate-particle and particle-particle potentials well depth energies, $\phi_{\mathbf{1}}$ and $\varphi_{1}$, respectively. These systems are drawn in Fig. 2. The measures adopted in Eq. (46) are given by

$V=\frac{4 \pi}{3}\left[Y R^{3}-(Y-1)(R-h)^{3} \Theta(R-h)\right]$,

$A=4 \pi\left[|Y| R^{2}+|Y-1|(R-h)^{2} \Theta(R-h)\right]$,

$\mathrm{J}=\partial_{R}^{2} V=8 \pi[Y R-(Y-1)(R-h) \Theta(R-h)]$,

$\mathrm{K}=\frac{1}{2} \partial_{R}^{2} A=4 \pi[|Y|+|Y-1| \Theta(R-h)]$.

which make sense for $h \ll R$. All these measures depend on the properties of the one-particle system, i.e., on the particlesubstrate potential parameters and $T$. In Eqs. (48) and (50) the dependence on $|Y-1|$ splits $A$ and $\mathrm{K}$ in two branches, one for the SW cavity $|Y-1|=Y-1$, and the other for the SS cavity $|Y-1|=-(Y-1)$. This is necessary to obtain the appropriate behavior for 2 -HS in a $\mathrm{HC}$ with a hard core. ${ }^{1} \mathrm{On}$ the other hand, although the dependence on $|Y|$ is artificial, it is needed for the discussion given in Sec. IV. For HS particles in a HC the Eqs. (47)-(50) reduce to the simple geometrical relations: $V=4 \pi R^{3} / 3, A=4 \pi R^{2}, j(R)=2 R^{-1}=\mathrm{J} / A$, and $k(R)=R^{-2}=\mathrm{K} / A$. The measures in Eqs. (47)-(50) can be also used for studying the $N$-interacting particle system even in the limit $N \rightarrow \infty$. Taking into account the set $\mathbf{M}$ defined in this work, the Eqs. (41) and (42) for $P$ and $\gamma$ are complemented with Eq. (43) in the following way:

$$
C_{\mathrm{J}}=\left.\frac{\partial F}{\partial \mathrm{J}}\right|_{T, \mathbf{M}-\mathrm{J}}, \quad C_{\mathrm{K}}=\left.\frac{\partial F}{\partial K}\right|_{T, \mathbf{M}-\mathrm{K}} .
$$

Herefrom we will focus on the SW cavity (further comments on the SS-cavity system are presented in the Appendix). For the specific case in which $\sigma>h$ and $R>h+\sigma^{\prime} / 2$ we derive analytic expressions for the coefficients $\left\{a_{2}, c_{2, \mathrm{~J}}, c_{2, \mathrm{~K}}\right\}$,

$$
\begin{aligned}
a_{2}= & \frac{\pi}{4|2 Y-1|}\left[\Sigma(4)-4 h^{2} Y(Y-1)\left(\Sigma(2)-\frac{h^{2}}{6}\right)\right], \\
c_{2, \mathrm{~J}}= & \frac{\pi h Y(Y-1)}{2(2 Y-1)}\left[\Sigma(4)-h^{2}\left(\Sigma(2)-\frac{h^{2}}{6}\right)\right], \\
c_{2, \mathrm{~K}}= & \frac{-\pi}{72|2 Y-1|}\left[\Sigma(6)+2 h^{2} Y(Y-1)\right. \\
& \left.\times\left(18 \Sigma(4)+9 h^{2} \Sigma(2)-h^{4}\right)\right], \\
\Sigma(n)= & X \sigma^{n}+(1-X) \sigma^{\prime n}=\sigma^{\prime n}-X\left(\sigma^{\prime n}-\sigma^{n}\right),
\end{aligned}
$$

where $b_{2}=-2 \pi \Sigma(3) / 3$. Note that $b_{2}$ is positive for HS and SS particles, but for SW particles it becomes negative at a certain critical value $X_{c}$. This value determines the Boyle's bulk temperature at which the two-body system behaves like a nearly ideal gas [see below the pressure in Eq. (56)]. It is interesting to mention that the $b_{2}$ cluster integral in $D$ dimension is proportional to $\Sigma(D)$, which implies that $a_{2}$ and $c_{2, \mathrm{~K}}$ for the inhomogeneous $2-\mathrm{SW}$ in a $\mathrm{HC}$ system are intrinsically linked to the properties of the SW system in $D=4$ and 6 . We may define several critical temperatures by $\Sigma(n)=\varsigma^{n}$ (with $\varsigma<\sigma^{\prime}$ a constant value), which implies that $k_{B} T_{c}(n, \varsigma)$ $=-\phi_{1} / \ln \left[\left(\sigma^{\prime n}-\varsigma^{n}\right) /\left(\sigma^{\prime n}-\sigma^{n}\right)\right]$. For example, we find $b_{2}=0$ at $T=T_{c}(3,0)$ and for a hard cavity $a_{2}=0$ at $T$ $=T_{c}(4,0)$. In general, all the $T_{c}(n, 0)$ temperatures are positive only for SW particles, being $T_{c}(n, 0)<T_{c}(n+1,0)$, but they are negative (and therefore unphysical) for the SS particles.

The coefficients $\left\{a_{2}, c_{2, \mathrm{~J}}, c_{2, \mathrm{~K}}\right\}$ are functions of the interaction potential parameters but they do not depend on the cavity size $R$, which only appears in the set $\mathbf{M}$ of thermodynamic measures. Similar expressions to those presented in Eqs. (52)-(54) are shown in the Appendix for the cases $\sigma^{\prime}>h>\sigma$ and $\sigma^{\prime}<h$. The coefficients $\left\{a_{2}, c_{2, \mathrm{~J}}, c_{2, \mathrm{~K}}\right\}$ are reduced to those of 2-HS by replacing $X=1$ or $\sigma^{\prime}=\sigma$, and to those of $\mathrm{HC}$ by setting $Y=1$. Once we adopted a set $\mathbf{M}$ of thermodynamic measures [see Eqs. (47)-(50)] and a procedure to decompose the partition function [see Eq. (62)], we may proceed to analyze the thermodynamic EOS of the system. ${ }^{1}$ We find

$$
\begin{aligned}
& \beta P=2 Z^{-1}\left(V-b_{2}\right), \\
& \beta \gamma=-2 Z^{-1} a_{2}, \\
& \beta C_{\mathrm{J}}=2 Z^{-1} c_{2, \mathrm{~J}}, \\
& \beta C_{\mathrm{K}}=2 Z^{-1} c_{2, \mathrm{~K}} .
\end{aligned}
$$

Here, the physical consequences of the null values in $b_{2}$ or $a_{2}$ are apparent. In particular, $a_{2}=0$ produces a quite similar thermodynamic behavior to that found in a quasihomogeneous system because $\gamma=0$. We remark that in a few-body inhomogeneous system a slightly different choice of $V$ modifies $P, P_{w}$, and also the other EOS. The intensivelike properties are balanced in a Laplace-like relation

$$
P-P_{w}=\gamma \frac{\mathrm{J}^{*}}{A^{*}}+C_{\mathrm{J}} \frac{\mathrm{K}^{*}}{A^{*}},
$$

with

$$
\beta P_{w}=2 Z^{-1}\left(V-b_{2}+a_{2} \frac{\mathrm{J}^{*}}{A^{*}}-c_{2, \mathrm{~J}} \frac{\mathrm{K}^{*}}{A^{*}}\right),
$$

where $A^{*}=\partial_{R} V, \quad \mathrm{~J}^{*}=\partial_{R} A$ and $2 \mathrm{~K}^{*}=\partial_{R} \mathrm{~J}$ reduce to the measures $A, \mathrm{~J}$, and $\mathrm{K}$ for the pure repulsive $\mathrm{SSC}$ potential. For a SWC potential the magnitudes in the above equations marked with an asterisk are different from the measures. We note that ratios such us $\mathrm{J}^{*} / A^{*}$ and $\mathrm{J} / A$ contain a smooth dependence on $T$, e.g., for large $R$ values $\mathrm{J}^{*} / A^{*}$ $\simeq 2(2 Y-1) R^{-1}>0$. Another simple expression for $P_{w}$ that 


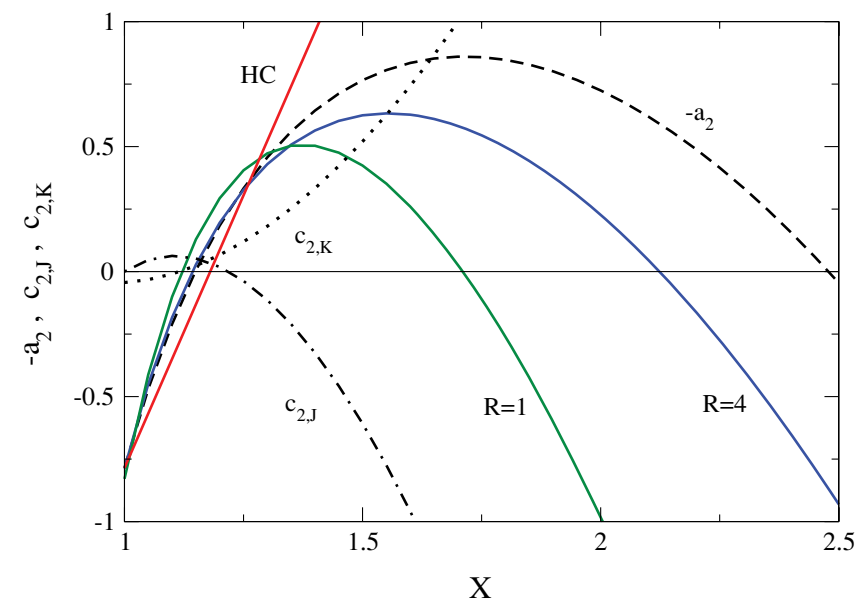

FIG. 3. The coefficients related to the thermodynamic properties of the 2SW-SWC system. $-a_{2}, c_{2, \mathrm{~J}}$, and $c_{2, \mathrm{~K}}$ are plotted in dashed, dot-dashed, and dotted lines, respectively. The dependence of $-a_{2}(R)$ on the radius is also shown in this plot: the dashed line corresponds to $-a_{2}(R \rightarrow \infty)$, while the continuous curves correspond to $-a_{2}(R=4)$ and $-a_{2}(R=1)$. The straight line represents the $-a_{2}$ coefficient for a HC [by setting $Y=1$ in Eq. (52)]. All the curves are also labeled for reference.

relates it to the density at the discontinuities of the substrate potential may be derived from Eq. (36). In Fig. 3 we show the typical behavior of $\left\{-a_{2}, c_{2, \mathrm{~J}}\right.$, and $\left.c_{2, \mathrm{~K}}\right\}$ related to the thermodynamic magnitudes $\left\{\beta \gamma, \beta C_{\mathrm{J}}\right.$, and $\left.\beta C_{\mathrm{K}}\right\}$. In this representation we set $Y=X$ and $h=\sigma^{\prime}-\sigma$, which means that substrate-particle and particle-particle potentials have a similar behavior. The plotted curves correspond to $\sigma=1$ and $h=0.6$. One important result shown in this plot is that the surface tension $\gamma$ depicted by $-a_{2}$, changes its sign twice, while the two curvature coefficients change their sign once. Besides, the $c_{2, J}$ coefficient is zero at $X=1$. It is worth noticing that the adopted set of measures as well as the decomposition rule strong influence the thermodynamic description of the system. Other plausible alternative could be the set of measures $\mathbf{M}=\{V, A, R\}$ along with the decomposition rule

$$
Z_{(1)} b_{2}(\text { pore })=V b_{2}-a_{2}(R) A,
$$

being $a_{2}(R)=a_{2}-c_{2, \mathrm{~J}} \mathrm{~J} / A-c_{2, \mathrm{~K}} \mathrm{~K} / A$. This new set of measures $\mathbf{M}=\{V, A, R\}$ is usually adopted to describe inhomogeneous fluids with spherical symmetry. Besides, a similar selection was adopted in Ref. 1 for the thermodynamic analysis of 2-HS confined to different simple geometrical cavities. The thermodynamic magnitudes of the system verify Eqs. (41) and (42) and

$$
C_{R}=\left.\frac{\partial F}{\partial R}\right|_{T, \mathbf{M}-R}
$$

The pressure $P$ is given in Eq. (56), while the other two EOS are

$$
\begin{aligned}
& \beta \gamma(R)=-2 Z^{-1} a_{2}(R), \\
& =\beta \gamma+\beta\left(C_{\mathrm{J}} \mathrm{J}+C_{\mathrm{K}} \mathrm{K}\right) / A \text {, } \\
& \beta C_{R}=-2 Z^{-1} A \partial_{R} a_{2}(R),
\end{aligned}
$$

where $\gamma(R)$ is the surface tension, which explicitly depends on the measure $R$. In Fig. 3 we illustrate $-a_{2}(R)$ for different values of $R$. There, it is clear that this coefficient varies smoothly with $R$. The Laplace-like balance reads

$$
P-P_{w}=\gamma(R) \frac{\mathrm{J}^{*}}{A^{*}}+C_{R} \frac{1}{A^{*}} .
$$

This expression and that in Eq. (60) are general and should be applicable to any fluid inside of a spherical cavity system. For the particular case of the 2-SW system, the Eq. (66) may be rewritten as

$P-P_{w}=\gamma(R)\left(\frac{\mathrm{J}^{*}}{A^{*}}-\frac{A}{A^{*}} \beta C_{R}\right)+\frac{A}{A^{*}} \frac{\partial \gamma(R)}{\partial R}$,

where the term $\beta C_{R}$ is of order $R^{-6}$ for large values of $R$. The first order dependence of the fluid-substrate surface tension on the curvature is given by the Tolman length

$$
\frac{\gamma(R)}{\gamma_{\infty}}-1=-\frac{c_{2, \mathrm{~J}}}{a_{2}} \frac{\mathrm{J}}{A}-\frac{c_{2, \mathrm{~K}}}{a_{2}} \frac{\mathrm{K}}{A}, \quad \delta_{\mathrm{Tol}}=-\frac{c_{2, \mathrm{~J}}}{a_{2}},
$$

where $\gamma_{\infty}$ is obtained by replacing $a_{2}(R) \rightarrow a_{2}$ in the explicit term of Eq. (64) (a further discussion related to this topic is presented in Ref. 1). In the limit where $R$ goes to infinity $\mathrm{J} / A \approx 2(2 Y-1)^{-1} R^{-1}>0$. Note that we adopted a convention which identifies $\delta_{\text {Tol }}$ with the coefficient of $\mathrm{J} / A$. From Fig. 3 it is clear that $a_{2}$ is zero at a certain temperature for which does not make sense to derive $\delta_{\mathrm{Tol}}$ for the 2SW-SWC system. On the other side, $\delta_{\mathrm{Tol}}$ is null for the case $h=0$ or $Y=1$, but not for $X=1$, or $\sigma^{\prime}=\sigma$. Taking into account this result, we expect a null fluid-substrate Tolman length, $\delta_{\mathrm{Tol}}=0$, for any two-particle system in a hard spherical cavity independently on the (finite-range) particle-particle potential.

\section{Adsorption and surface of tension}

We extend our analysis of the confined two-body system by studying the surface adsorption and the spherical fluidsubstrate interface. In order to make this, we define the substrate adsorption $\Gamma$ with respect to the central density $\rho_{h}(0)$ under the action of the substrate potential as

$$
N_{A}=\Gamma A \equiv 4 \pi \int_{0}^{\infty}\left[\rho(r)-e_{1}(r) \rho_{h}(0)\right] r^{2} d r
$$

where $N_{A}$ represents the mean number of particles adsorbed on the surface. Equation (69) can be written as

$$
\frac{N_{A}}{V}=\bar{\rho}-\rho_{h}(0) \text {. }
$$

In the case of the two-body system analyzed above, using Eqs. (26) and (64) we obtain $\rho_{h}(0)=2 Z^{-1}\left(Z_{(1)}-2 b_{2}\right)$ and

$$
\frac{\Gamma}{N}=-\beta \gamma(R),
$$

which we expect will also be valid for other few-body systems with $N \gtrsim 2$. By combining Eqs. (70) and (71) we find the following interesting relation:

$$
\frac{\rho_{h}(0)}{\bar{\rho}}-1=\beta A \gamma(R) \text {. }
$$


Equations (71) and (72) trace exact relations between adsorption, surface tension, and one-body density distribution for a 2-SW-SWC system. These equations allow us to relate $A \gamma(R) \ll 0$ to the existence of an adsorbed fluid film on the substrate surface and $A \gamma(R) \gg 0$ to an excess of the amount of fluid in the inner part of the cavity. A similar behavior in the context of many-body (macroscopic) systems appears in the capillary condensation phenomena. ${ }^{15}$

In addition, to study the substrate-fluid spherical interface we take a point of view that is customary to adopt in the analysis of the spherical liquid-vapor free interface. First, we are interested in exploring a magnitude similar to the liquidvapor equimolar radius $R_{e}$, which for a spherically confined fluid may be defined by

$$
N=4 \pi \int_{0}^{R_{e}} e_{1}(r) \rho_{h}(0) r^{2} d r .
$$

Here $V\left(R_{e}\right)=4 \pi \int_{0}^{R_{e}} e_{1}(r) r^{2} d r \leq V$ corresponds to the equimolar volume. This definition for $R_{e}$ implies that $0<R_{e}<R$ and shows that the equimolar radius is not defined in the range where $\bar{\rho}>\rho_{h}(0)$. We can formulate an alternative definition for $R_{e}$ that solves this problem. We assume that $\Delta=R_{e}-R$ is a small magnitude and define

$$
N=V \rho_{h}(0)+\Delta A \rho_{h}(0),
$$

that is similar to a truncated series expansion of Eq. (73). It may be transformed to

$$
\Delta=\frac{V}{A}\left(\frac{\bar{\rho}}{\rho_{h}(0)}-1\right)=\frac{\Gamma}{\rho_{h}(0)},
$$

where the last equality comes from Eq. (70). It is apparent that $R_{e}<R$ only for positive adsorption [or negative $\gamma(R)$ ] and that $\Delta$ may not be a small magnitude. Finally, we are interested in determining the location of the surface of tension, that is, the locus of a geometrical surface where the surface tension is supposed to act. To make this we adopt the measures $\mathbf{M}=\left\{V, A_{s}, R, R_{s}\right\}$ where the subscript $s$ indicates the geometrical magnitudes related to the surface of tension, i.e., $A_{s}=4 \pi R_{s}^{2}$. Additionally, we adopt the decomposition rule

$$
Z_{(1)} b_{2}(\text { pore })=V b_{2}-a_{2}\left(R, R_{s}\right) A_{s},
$$

with $a_{2}\left(R, R_{s}\right)=a_{2}(R) A / A_{s}$. For the surface $A_{s}$, the original form of the Laplace equation is supposed to be valid, therefore, we consider

$$
P-P_{w}=\gamma\left(R, R_{s}\right) \frac{\mathrm{J}_{s}}{A^{*}} .
$$

The missing term in Eq. (77) with the $C_{R}+C_{R_{s}}$ contribution is identically zero. We identify $C_{R_{s}}$ with the virtual work necessary to modify the position of the surface of tension at constant $\left\{V, A_{s}\right.$, and $\left.R\right\}$. The system EOS are given by Eq. (56) and

$$
\gamma\left(R, R_{s}\right)=\gamma(R) A / A_{s},
$$

where $\gamma\left(R, R_{s}\right)$ is the surface tension exerted on the surface of tension with the following constraint

$$
\beta \gamma(R)\left[\mathrm{J}^{*}-\frac{\mathrm{J}_{s} A}{A_{s}}\right]+\beta C_{R}=0,
$$

which defines the locus of the surface of tension. Using Eqs. (64) and (65), the location of the surface of tension is given by

$$
\begin{aligned}
& R_{s}=2 A \gamma(R)\left[\mathrm{J}^{*} \gamma(R)+C_{R}\right]^{-1}, \\
& R_{s} \simeq \frac{2 A}{\mathrm{~J} *}+2\left(\frac{\mathrm{J}}{\mathrm{J} *}-2 \frac{A \mathrm{~K}^{*}}{\mathrm{~J} * 2}\right) \frac{c_{2, \mathrm{~J}}}{a_{2}},
\end{aligned}
$$

where the last expression shows the linear dependence of $R_{s}$ on the Tolman length.

\section{PROPERTIES OF OTHER SW SYSTEMS}

There is a second two-body system strongly related to the 2-SW-SWC that is also analytically tractable. This is the 2-SW particles outside a square-well spherical obstacle (2SW-SWO) where the obstacle behaves like a third SW particle with fixed position, hard repulsion radius $R=R^{\prime}+\sigma / 2$, and a square well extended in the range $(R, R+h)$. The $\mathrm{CI}$ of such a system can be graphically decomposed [see Eqs. (2.a) and (2.b) in Ref. 1, related to Eq. (44) and Eqs. (44) and (62) in the current work]. The first term in Eqs. (44) and (46) is easily evaluated for both systems, 2-SW-SWC and 2SW-SWO. Besides, the term $Z_{(1)} b_{2}$ (pore) $-V b_{2}$ for the 2SW-SWC and the 2-SW-SWO systems are strongly related to each other. This allow us to obtain an analytic expression for the CI of 2-SW-SWO. We find that a simple mapping of this term from one system to the other is obtained by replacing the parameters $Y \rightarrow 1-Y$ and $R \rightarrow R+h$. The latter replacement is purely notational and is a consequence of referring $\mathrm{R}$ to the minimum allowed distance between a particle and the spherical obstacle. We may adopt the decomposition given in Eqs. (44) and (46) for 2-SW-SWO along with the following measures for the SW-SWO system

$$
\begin{aligned}
& V=V_{\infty}-\frac{4 \pi}{3}\left[Y R^{3}-(Y-1)(R+h)^{3}\right], \\
& A=4 \pi\left[|Y| R^{2}+|Y-1|(R+h)^{2}\right], \\
& \mathrm{J}=-8 \pi[Y R-(Y-1)(R+h)], \\
& \mathrm{K}=4 \pi[|Y|+|Y-1|],
\end{aligned}
$$

where $V_{\infty}$ is the infinite volume of the three dimensional euclidean space. The comparison between Eqs. (47)-(50) and Eqs. (82)-(85) shows an extra minus sign in J. This sign, which in the HS limit $(Y \rightarrow 1)$ produces a negative mean curvature $\mathrm{J}$, does not have its origin in the replacements $Y \rightarrow 1-Y$ and $R \rightarrow R+h$. The replacement of $Y$ by $1-Y$ only modifies slightly the coefficients of Eqs. (52)-(54) introducing a minus sign in $c_{2, \mathrm{~J}}$ in comparison with the expression given in Eq. (53). However, we adopt a convention that leaves $c_{2, \mathrm{~J}}$ unmodified by assigning this extra minus term to $J$ [note that this sign was already included in Eq. (84)]. On the other side, $A^{*}$ and $\mathrm{K}^{*}$ change their sign, but $\mathrm{J}^{*}$ does not change. In this context, the thermodynamic EOSs are given by 
Eqs. (56)-(59). The Laplace-like relation is still given by Eq. (60) and can be rewritten as

$$
P_{w}-P=\gamma \frac{\left(-\mathrm{J}^{*}\right)}{A^{*}}-C_{\mathrm{J}} \frac{\mathrm{K}^{*}}{A^{*}} .
$$

Here, $\mathrm{J}^{*}$ is positive but $A^{*}$ is essentially a negative magnitude (in the limit where $R$ goes to infinity $\mathrm{J}^{*} / A^{*} \propto-2 R^{-1}<0$ ). To study the 2-SW-SWO we select the second option for the set of measures and the decomposition rule [see Eq. (62)]. We found Eqs. (56), (64), and (65) which leads to the following Laplace-like relation

$$
\begin{aligned}
P_{w}-P & =\gamma \frac{\left(-\mathrm{J}^{*}\right)}{A^{*}}-C_{R} \frac{1}{A^{*}} \\
& =\gamma\left(-\frac{\mathrm{J}^{*}}{A^{*}}+\frac{A}{A^{*}} \beta C_{R}\right)+\frac{A}{A^{*}} \frac{\partial \gamma}{\partial R},
\end{aligned}
$$

where $A / A^{*}$ is essentially a negative magnitude (for large $R$ values $A / A^{*} \approx-1$ ). If we do not take into account the term $\beta C_{R}$, the Eq. (87) results very similar to Eq. (1) in Ref. 10, which is supposed to be valid for any fluid surrounding a spherical shaped hard wall. In addition, from Eq. (87) we may recover Eq. (68) and obtain the curvature dependence of the substrate-fluid surface tension. Note that now $J / A$ is essentially negative (in the limit where $R$ goes to infinity $\mathrm{J} / A \propto-2 R^{-1}<0$ ). Therefore, keeping the same convention for the sign of $\delta_{\mathrm{Tol}}$ as in Eq. (68) we still find

$$
\delta_{\mathrm{Tol}}=-\frac{c_{2, \mathrm{~J}}}{a_{2}},
$$

where $c_{2, J}$ is given by Eq. (53).

It is interesting to make a brief digression about the consequences of the above mentioned results for the 2-SW-SWC and 2-SW-SWO on the inhomogeneous systems of many SW particles. As it has recently been discussed in Ref. 1, some properties of the system of $\mathrm{N}$-SW particles and the equivalent open system of many-SW particles may be evaluated in the low density limit. The core of the relation between the twobody systems and the many-body systems is given in Eq. (44). Since we analytically evaluated $Z$ for the two-body system we obtained an expression for $b_{2}$ (pore), which is a central quantity in the virial expansion theory of real gases. For instance, the Eq. (57) analyzed in the context of the $N$-particles system transforms to

$$
\beta \gamma=-\frac{N(N-1)}{V^{2}} a_{2}+O\left(V^{-3}\right) \approx-\rho^{2} a_{2},
$$

for a large enough radius $R$. Since Eq. (89) does not involve any shape dependence it also applies to any surface, independently of its shape. Based on these ideas we can derive the many-body version of Eq. (68),

$$
\begin{aligned}
\frac{\gamma}{\gamma_{\infty}}-1 & =-\frac{c_{2, \mathrm{~J}}}{a_{2}} \frac{\mathrm{J}}{A}-\frac{c_{2, \mathrm{~K}}}{a_{2}} \frac{\mathrm{K}}{A}+O\left(V^{-1}\right), \\
\delta_{\mathrm{Tol}} & =-\frac{c_{2, \mathrm{~J}}}{a_{2}}+O\left(V^{-1}\right) .
\end{aligned}
$$

We thus obtained the first order analytical dependence on density of $\delta_{\text {Tol }}$ (its dependence to zero order) for a system of SW interacting particles in contact with a SW spherical substrate. Even though the interacting potential analyzed here is a crude

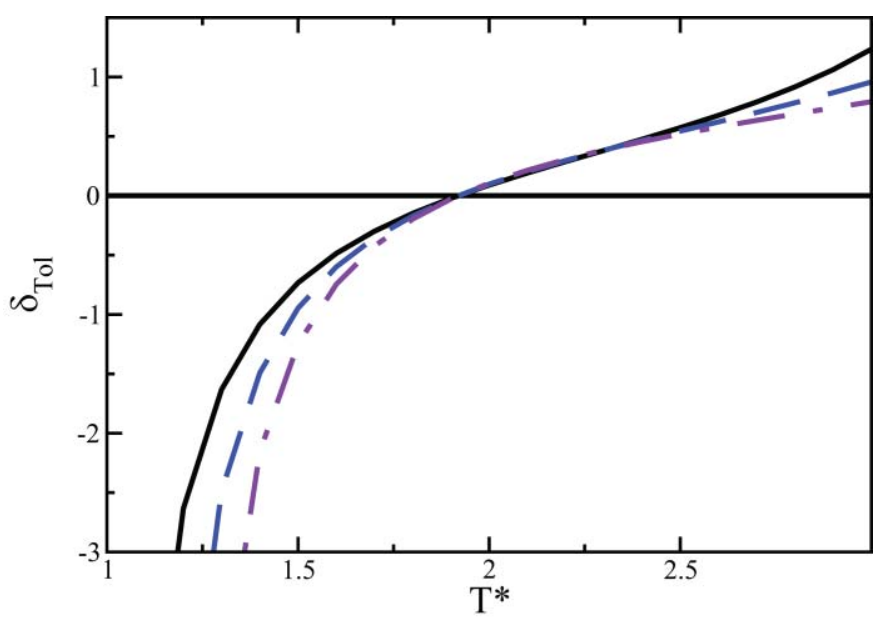

FIG. 4. The Tolman length $\delta_{\mathrm{Tol}}=-c_{2, \mathrm{~J}} / a_{2}$ as a function of $T^{*}=k_{B} T / \phi$. From left to right in the bottom of the figure we plot for the values $\varphi / \phi=$ $0.75,1.00$, and 1.5. The HC curve coincides with the zero ordinate line.

sketch of a real particle-particle and particle-substrate potentials, it retains the attractive well that is a relevant characteristic of real potentials. In this sense we should expect the behavior of $\delta_{\mathrm{Tol}}$ for the case of more realistic potentials to be similar to that obtained for SW. In Fig. 4 we plot the $\delta_{\mathrm{Tol}}$ function for the 2-SW-SWC (and 2-SW-SWO) system and the first order term of $\delta_{\text {Tol }}$ for the many-SW-SWC (and many-SW-SWO) system as a function of the temperature, assuming $\sigma=1, \sigma^{\prime}=1.3$, and $h=0.8$. It is clear from the plot that the root of $\delta_{\text {Tol }}$ only depends on the length parameters $\sigma$, $\sigma^{\prime}$, and $h$, but not on the energy parameters $\varphi$ and $\phi$. In the analysis of the $N$-SS and many-SS systems we also obtained the same Eqs. (89) and (90).

The major contribution of this section is that we present a method to relate the 2-SW-SWC system to the 2-SW-SWO system, as well as to the many-SW-SWC and the many-SWSWO systems. The inclusion in our discussion of the SS particles, the SS cavity, and the SS obstacle is straightforward and follows the treatment derived for the 2-SW-SWC system.

\section{FINAL REMARKS}

The most important result presented in this work is the analytical expression of the canonical partition function for a two-body system of interacting or thermal particles in a spherical cavity, which exerts a nonhard interaction potential. In our study we analyzed potentials that are piecewise constant defined and include the square-well and the squareshoulder potentials. We found that the CI of this inhomogeneous system, succinctly given in Eq. (19), is polynomial in each analytic branch. In addition, we obtain analytical expressions for other relevant magnitudes of the system such as the one-body density distribution $\rho(\mathbf{r})$ and an averaged two-body density distribution $\bar{g}(\mathbf{r})$. We developed a method that allows for making a thermodynamic description of few-body inhomogeneous systems by extending the use of thermodynamic measures to nonhard interactions. This method was used to investigate the two-body system in order to obtain the EOS, 
the pressure-for-work, the inner pressure, the surface tension, and the two curvature-work terms. We also derived a relation between the EOS, which is similar to the Laplace equation that states the balance in the difference of pressures in a liquid droplet. However, as the obtained thermodynamic properties are strongly influenced by the adopted set of measures we complemented our analysis by studying the same two-body system with a second different set of thermodynamic measures. This study explicitly shows how the curvature of the substrate surface influences the surface tension and allow us to obtain an analytical expression for the substrate-fluid Tolman length. Additionally, we analyzed the adsorption in the spherical substrate, the radius of the equimolar density as well as the position of the surface of tension and demonstrated that it is possible to make a full study of this spherical inhomogeneous system.

The CI for the confined two-body system was used to derive the analytical expression of the CI for a system composed of two particles outside a spherical cavity. We extended this treatment to the inhomogeneous many-body system of square-well particles inside a spherical confinement as well as outside a spherical obstacle, in the low density limit. In such conditions we obtained an expression for the first density and curvature dependence of the surface tension along with an expression for the Tolman length. Based on our analysis we conclude that the Tolman length for systems, in a cavity and outside a spherical object, contains the same non-null zero order term in the density. This term has a null value for hard spherical substrates.

The studied square-well potential may be considered as a simplification that still retains the main characteristics of other more realistic potentials. Therefore, it is expected that for systems of particles with more realistic finite-range particle-particle interaction in contact with hard spherical substrates the Tolman length to be null to zero order in density.

\section{ACKNOWLEDGMENTS}

This research has funded by Argentina Grants UBACyT A023, UBACyT x099, ANPCyT-PICT 2008-0795, and ANPCyT-PICT 2006-00492.

\section{APPENDIX: EXPLICIT FORMS OF THE $a_{2}, c_{2, \mathrm{~J}}$, AND $c_{2, \mathrm{~K}}$ COEFFICIENTS FOR DIFFERENT CONDITIONS}

In what follows we present expressions for the coefficients in $Z_{(1)} b_{2}$ (pore) taken into account the decomposition given in Eq. (46) for the SWC with $R>3 h / 2$ and $R>h$ $+\sigma^{\prime} / 2$ : (i) for the case $0<h<\sigma$ see Eqs. (52)-(54), (ii) for the case $\sigma<h<\sigma^{\prime}$,

$$
\begin{aligned}
& a_{2}=\frac{\pi}{4|2 Y-1|}\left\{\Sigma(4)-4 Y(Y-1)\left[h^{2}(1-X)\left(\sigma^{2}-\frac{h^{2}}{6}\right)+\frac{1}{2} X \sigma^{3}\left(\frac{8}{3} h-\sigma\right)\right]\right\}, \\
& c_{2, \mathrm{~J}}=\frac{h \pi(Y-1) Y}{2(2 Y-1)}\left[\Sigma(4)-h^{2}(X-1)\left(\sigma^{\prime 2}-\frac{h^{2}}{6}\right)+\frac{1}{2} X \sigma^{3}\left(\frac{8}{3} h-\sigma\right)\right], \\
& c_{2, \mathrm{~K}}=\frac{-\pi}{72|2 Y-1|}\left\{\Sigma(6)+36 h^{2} \Sigma(4)+2 Y(Y-1)\left[-h^{4}(X-1)\left(h^{2}+9 \sigma^{\prime 2}\right)+X \sigma^{3}\left(16 h^{3}-9 h^{2} \sigma+\sigma^{3}\right)\right]\right\},
\end{aligned}
$$

and (iii) for the case $\sigma^{\prime}<h$,

$$
\begin{aligned}
a_{2}= & \frac{\pi}{4|2 Y-1|}[\Sigma(4)-2(Y-1) Y \\
& \left.\times\left(\frac{8}{3} h \Sigma(3)-\Sigma(4)\right)\right] \\
c_{2, \mathrm{~J}}= & \frac{h \pi Y(Y-1)}{4(2 Y-1)}\left(\frac{8}{3} h \Sigma(3)+\Sigma(4)\right) \\
c_{2, \mathrm{~K}}= & -\frac{\pi}{72|2 Y-1|}\{\Sigma(6)+2 Y(Y-1)[\Sigma(6) \\
& \left.\left.+h^{2}(16 h \Sigma(3)+9 \Sigma(4))\right]\right\}
\end{aligned}
$$

On the other side, for the SSC with $R>3 h / 2$ and $R>h$ $+\sigma^{\prime} / 2$, those coefficients are given by: (i) for the case $0<h$ $<\sigma$

$$
\begin{gathered}
a_{2}=\frac{\pi}{4}\left[\Sigma(4)+4 h^{2} Y(1-Y)\left(\Sigma(2)-\frac{h^{2}}{6}\right)\right], \\
c_{2, \mathrm{~J}}=\frac{\pi}{2} h^{3} Y(1-Y)(1-2 Y)\left(\Sigma(2)-\frac{h^{2}}{6}\right), \\
c_{2, \mathrm{~K}}=-\frac{\pi}{72}\left\{\Sigma(6)+2 h^{4}(1-Y) Y\left[-9 \Sigma(2)+h^{2}\right.\right. \\
\left.\left.+64(1-Y) Y\left(\Sigma(2)-\frac{h^{2}}{6}\right)\right]\right\},
\end{gathered}
$$


(ii) for $\sigma<h<\sigma^{\prime}$,

$$
\begin{aligned}
a_{2}= & \frac{\pi}{4}\left\{\Sigma(4)+2 Y(1-Y)\left[2 h^{2}(1-X)\left(\sigma^{2}-\frac{h^{2}}{6}\right)+\sigma^{3} X\left(\frac{8}{3} h-\sigma\right)\right]\right\}, \\
c_{2, \mathrm{~J}}= & \frac{\pi}{4} h Y(1-Y)(1-2 Y)\left[2 h^{2}(1-X)\left(\sigma^{\prime 2}-\frac{h^{2}}{6}\right)+\sigma^{3} X\left(\frac{8}{3} h-\sigma\right)\right], \\
c_{2, \mathrm{~K}}= & -\frac{\pi}{72}\left\{\Sigma(6)+2 h^{4} Y(1-Y)(1-X)\left[h^{2}-9 \sigma^{\prime 2}+12 Y(1-Y)\left(6 \sigma^{\prime 2}-h^{2}\right)\right]\right. \\
& \left.+2 \sigma^{3} Y(1-Y) X\left[-h^{2}(16 h-9 \sigma)-\sigma^{3}+12 h^{2} Y(1-Y)(8 h-3 \sigma)\right]\right\},
\end{aligned}
$$

and (iii) for $\sigma^{\prime}<h$,

$$
\begin{aligned}
a_{2}=\frac{\pi}{4} & {\left[\Sigma(4)+2 Y(1-Y)\left(\frac{8}{3} h \Sigma(3)-\Sigma(4)\right)\right], } \\
c_{2, \mathrm{~J}}= & \frac{\pi}{4} h Y(1-Y)(1-2 Y)\left(\frac{8}{3} h \Sigma(3)-\Sigma(4)\right), \\
c_{2, \mathrm{~K}}= & \frac{\pi}{72}\left\{-\Sigma(6)+2 Y(1-Y)\left[16 h^{3}(1-6 Y(1-Y))\right.\right. \\
& \left.\left.\times \Sigma(3)-9 h^{2}(1-2 Y)^{2} \Sigma(4)+\Sigma(6)\right]\right\}
\end{aligned}
$$

$\Sigma(n)=X \sigma^{n}+(1-X) \sigma^{\prime n}$.

$$
\begin{aligned}
& a_{2}=|2 Y-1|^{-1}\left(a_{2,0}-\Delta a_{2}\right), \\
& c_{2, \mathrm{~J}}=h(2 Y-1)^{-1}\left(2 Y(Y-1) a_{2,0}-\frac{1}{2} \Delta a_{2}\right) .
\end{aligned}
$$

For the case of a SSC we find

$$
\begin{aligned}
& a_{2}=a_{2,0}-\Delta a_{2}, \\
& c_{2, \mathrm{~J}}=\frac{1}{2} h(2 Y-1) \Delta a_{2} .
\end{aligned}
$$

Finally,

$$
\Delta a_{2}=\pi Y(Y-1) \times \begin{cases}h^{2}\left(\Sigma(2)-h^{2} / 6\right), & \text { if } h \leq \sigma \leq \sigma^{\prime} \\ h^{2}(1-X)\left(\sigma^{\prime 2}-h^{2} / 6\right)+\sigma^{3} X(4 / 3 h-1 / 2 \sigma), & \text { if } \sigma \leq h \leq \sigma^{\prime} \\ 4 / 3 h \Sigma(3)-1 / 2 \Sigma(4), & \text { if } \sigma \leq \sigma^{\prime} \leq h\end{cases}
$$

${ }^{1}$ I. Urrutia, J. Chem. Phys. 133, 04503 (2010); e-print arXiv:1005.0723.

${ }^{2}$ I. Urrutia, J. Stat. Phys. 131, 597 (2008); e-print arXiv:condmat/0609608.

${ }^{3}$ I. Urrutia and L. Szybisz, J. Math. Phys. 51, 033303 (2010); e-print arXiv:0909.0246.

${ }^{4}$ J. O. Hirschfelder, C. F. Curtis, and R. B. Bird, Molecular Theory of Gases and Liquids (John Wiley \& Sons, New York, 1954).

${ }^{5}$ D. A. McQuarrie, Statistical Mechanics (University Science Books, Sausalito, 2000).

${ }^{6}$ R. López-Rendón, Y. Reyes, and P. Orea, J. Chem. Phys. 125, 84508 (2006).

${ }^{7}$ R. Espíndola-Heredia, F. del Río, and A. Malijevsky, J. Chem. Phys. 130, 024509 (2009).
${ }^{8}$ P. J. Lu, E. Zaccarelli, F. Ciulla, A. B. Schofield, F. Sciortino, and D. A. Weitz, Nature (London) 453, 499 (2008).

${ }^{9}$ S.-C. Kim and T. Munakata, J. Korean Phys. Soc. 43, 997 (2003).

${ }^{10}$ E. M. Blokhuis and J. Kuipers, J. Chem. Phys. 126, 054702 (2007).

${ }^{11}$ Y. He, J. Mi, and C. Zhong, J. Phys. Chem. B 112, 7251 (2008).

${ }^{12}$ D. A. McQuarrie and J. S. Rowlinson, Mol. Phys. 60, 977 (1987).

${ }^{13}$ T. L. Hill, Statistical Mechanics (Dover, New York, 1956).

${ }^{14}$ T. L. Hill, Thermodynamics of Small Systems (Dover, New York, 1994).

${ }^{15}$ E. Charlaix and M. Ciccotti, in Handbook of Nanophysics: Principles and Methods, edited by K. D. Sattler (CRC, Boca Raton, 2010), Vol. 1, Chap. 12; e-print arXiv:0910.4626. 